\title{
Monoclonal antibody Py recognizes neurofilament heavy chain and is a selective marker for large diameter neurons in the brain
}

\author{
Heidi R Fuller ${ }^{1,2}$, Lucia Marani ${ }^{3}$, Ian Holt ${ }^{1,2}$, Peter L Woodhams ${ }^{4}$, Michael M Webb ${ }^{5}$ and \\ Monte A Gates ${ }^{2 *}$ \\ ${ }^{1}$ Wolfson Centre for Inherited Neuromuscular Disease, RJAH Orthopaedic Hospital, \\ Oswestry, SY10 7AG, UK; ${ }^{2}$ Institute for Science and Technology in Medicine, Keele \\ University, Staffordshire, ST5 5BG, UK; ${ }^{3}$ School of Sports, Exercise and Health Sciences, \\ Loughborough University, Loughborough LE113TU, UK; ${ }^{4}$ Emeritus; ${ }^{5}$ Mitobridge Inc, 1030 \\ Massachusetts Avenue, Cambridge, MA 02138, USA
}

*Corresponding author

Email: m.a.gates@keele.ac.uk

Telephone: $+44(0) 1782733875$

Acknowledgments: We would like to thank Prof. Glenn E Morris for his invaluable insights into the growth of the Py clones, and the production of antibodies for immunofluorescence. This research did not receive any specific grant from funding agencies in the public, commercial, or not-for-profit sectors. 


\section{$\underline{\text { Abstract }}$}

Almost 30 years ago, the monoclonal antibody Py was developed to detect pyramidal neurons in the CA3 region of the rat hippocampus. The utility of this antibody quickly expanded when several groups discovered that it could be used to identify very specific populations of neurons in the normal, developing, and diseased or injured central nervous system. Despite this body of literature, the identity of the antigen that the Py antibody recognizes remained elusive. Here, immunoprecipitation experiments from the adult rat cortex identified the Py antigen as neurofilament heavy chain (NF-H). Double immunolabelling of sections through the rat brain using Py and NF-H antibodies confirmed the identity of the Py antigen, and reveal that $\mathrm{Py} / \mathrm{NF}-\mathrm{H}+$ neurons appear to share the feature of being particularly large in diameter. These include the neurons of the gigantocellular reticular formation, pyramidal neurons of layers II/III and V of the cortex, cerebellar Purkinje neurons as well as CA3 pyramidal neurons. Taken together, this finding gives clarity to past work using the monoclonal Py antibody, and immediately expands our understanding of the importance of NF-H in neural development, functioning, and disease.

Keywords: monoclonal antibody Py; neurofilament heavy chain; large diameter neuron; neurodegenerative disease 


\section{$\underline{\text { Introduction }}$}

In 1989, a study by Woodhams et al. (1989) detailed the production and characterization of a new antibody that labelled a subset of neurons in the hippocampus, cortex, cerebellum and brain stem. Because the antibody was useful for distinguishing pyramidal neurons from the CA3 region of the hippocampus both in vivo and in vitro, they called the monoclonal IgM antibody "Py". The usefulness of this antibody was almost immediately realized, as Raisman and colleagues used it to identify and detail CA3 hippocampal transplants to adult rats (Field et al., 1991), and Whittemore and colleagues that the Py antigen was expressed by immortalized stem cells transplanted (and incorporating) into the CA3 region of the rat (Shihabuddin et al., 1996). Subsequent work by Brook et al. (1997a and 1998) found that the antibody stained neurons along the spinal column, most notably motor neurons, and the neurons of Clarke's nucleus. Additional work by Houweling et al. (1999) found that Py labelled neurons in the developing spinal cord at very early stages of development (i.e., embryonic day 15). The antibody further proved useful for highlighting important changes in anatomical structures of the brain and spinal cord in response to gene alterations, injury or disease models. Py, for example, was used to highlight cortical neurons affected by deletion of the mouse-enabled gene, which displays midline crossing defects in the corpus callosum (Lanier et al., 1999). In the red nucleus and Clarke's nucleus, Py highlighted a loss of cellular content after axotomy of the rubrospinal and spinocerebellar tracts, respectively (Brook et al., 1997b and 1998). In a transgenic mouse model of amyotrophic lateral sclerosis (G93A), Py was shown useful for detecting early changes in motoneurons before the onset of neuronal cell death in the spinal cord (Joosten et al., 2001).

However, the identity of the antigen that the Py antibody recognises has, for almost 30 years, remained elusive. Determining the antigen recognised by the Py antibody would be beneficial not only for understanding the findings of past work that has used the antibody, but may also allow for some understanding of what the function of such a protein might be for neurons that express the antigen in the developing and adult central nervous system (CNS). Here, immunoprecipitation experiments from the adult rat cortex have identified the Py antigen as neurofilament heavy chain (NF-H). This finding was confirmed using double-label immunofluorescence analysis in sections through the rat brain, which highlighted its expression in a subset of neurons in the cortex, hippocampus, septum, basal forebrain, brain stem and cerebellum. Coupling findings from studies using the Py antibody with studies 
using the NF-H antibody should enhance our understanding of the role that NF-H plays in the development, maturation, and diseased states of the mammalian CNS.

\section{Methods}

\section{Py antibody production}

A Py IgM hybridoma cell line (2011 clone) was quickly thawed in a $37^{\circ} \mathrm{C}$ water bath for 1 minute. The cell suspension was subsequently centrifuged at $300 \mathrm{~g}$ for 5 minutes and the supernatant discarded. The cells were suspended in a hybridoma medium (DMEM, 20\% horse serum, $1 \%$ non-essential amino acids, $1 \%$ glutamax, $1 \%$ penicillin streptomycin fungizone) and centrifuged again at $300 \mathrm{~g}$ for 5 minutes and cultured in a T25 flask at $37^{\circ} \mathrm{C}$ in $5 \% \mathrm{CO} 2$. The cells were allowed to expand for 1 week, and were subsequently split 1:3 into T75 flasks filled with hybridoma medium. The cells were grown at $37^{\circ} \mathrm{C}$ for an additional 2 weeks, when the supernatant was collected for use in immunofluorescence staining. The antibody was stored until use at $-80^{\circ} \mathrm{C}$ in aliquots to minimise freeze thawing cycles.

\section{Tissue extraction}

All procedures were approved by the Animal Welfare \& Ethical Review Body (AWERB) at Keele University, and were carried out under the licensed authority of the UK Home Office (PPL40/3556). Adult, female Sprague Dawley rats ( 230g weight) were given an overdose of pentobarbitone anesthetic $(0.5 \mathrm{ml} / 100 \mathrm{~g})$ via an intraperitoneal injection, and transcardially perfused with ice-cold sterile $0.9 \%$ sodium chloride. The brains were quickly removed and layers II - V of the cortex (i.e., areas which are known to express the Py antigen, based upon immunofluorescence observations) were dissected from the dorsal region above the striatum. Cortical tissue was placed on a small spatula and excess saline solution removed before placing the sample into a $1.5 \mathrm{ml}$ Eppendorf tube. The dissected tissue was homogenised in approximately four volumes (w/v) of modified RIPA buffer (1\% NP40, 0.25\% deoxycholate, $1 \mathrm{mM}$ EDTA, $150 \mathrm{mM} \mathrm{NaCl}$, in $50 \mathrm{mM}$ Tris-HCl, $\mathrm{pH} 7.4$ ) using a small pellet pestle. The extracts were sonicated for 5 seconds, left on ice for 10 minutes, followed by centrifugation at $13,000 \mathrm{~g}$ for 10 minutes at $4^{\circ} \mathrm{C}$ to pellet any insoluble material.

\section{Immunoprecipitation}


For immunoprecipitation with anti-NF-H: anti-mouse Pan Ig-coated magnetic beads $(50 \mu \mathrm{l})$ (Dynal, Oslo) were washed three times with 4\% BSA in PBS (using a magnetic collector to separate the beads from solution). The beads were then incubated with a monoclonal antibody that recognises phosphorylated and non-phosphorylated forms of NF-H (Sigma; N0142) ( $50 \mu \mathrm{l}$ of a $1 / 50$ dilution) for 30 minutes at room temperature with gentle rolling. The beads were then washed three times with PBS $(200 \mu 1)$, followed by incubation for 1 hour at room temperature with the rat cortex RIPA extract $(75 \mu l)$ on a roller. The unbound material was carefully removed from the beads and stored at $-80^{\circ} \mathrm{C}$ until use. The beads were washed six times with RIPA buffer $(200 \mu \mathrm{l})$ before eluting captured material by heating at $90^{\circ} \mathrm{C}$ for 3 minutes in $2 \times$ SDS sample buffer $(75 \mu l)$ (2\% sodium dodecyl sulphate- SDS, 5\% 2mercaptoethanol, $62.5 \mathrm{mM}$ Tris- $\mathrm{HCl}, \mathrm{pH}$ 6.8). The same method was used for immunoprecipitation with the Py monoclonal antibody $(50 \mu 1$ neat), except that a Protein $\mathrm{L}$ matrix (50 $\mu$ l) (CBind L, Sigma) was used for antibody capture instead of Pan Ig magnetic beads because the Py MAb is IgM class.

\section{Western blotting}

Protein samples were heated at $90^{\circ} \mathrm{C}$ in SDS sample buffer (2\% SDS, 5\% 2-mercaptoethanol 62.5mM Tris-HCl, $\mathrm{pH}$ 6.8) for 3 minutes, separated on 4-12\% SDS-PAGE gradient gel (ThermoFisher Scientific), followed by transfer to nitrocellulose membrane over-night. After blocking non-specific sites with $4 \%$ powdered milk solution, membranes were incubated with either Py MAb (1/10), NF-H MAb (Sigma; N0142) (1/1000) or SMI-32 MAb (EMD Millipore; NE1023) (1/1000) diluted in dilution buffer (PBS, 1\% fetal bovine serum, $1 \%$ horse serum and $0.1 \% \mathrm{BSA}$ ). Antibody reacting bands were visualized by development with peroxidase-labelled rabbit anti-mouse IgG $(1 \mu \mathrm{g} / \mathrm{ml}$ in dilution buffer; Dako) (for NF-H and SMI-32) or peroxidase-labelled goat anti-mouse IgM (1/2000; Jackson ImmunoResearch) (for Py), and a chemiluminescent detection system (West Pico, Pierce).

To determine whether the epitope recognized by the Py antibody is glycosylated, electroblotted membranes were subject to sodium metaperiodate treatment to destroy pentose and hexose carbohydrate epitopes by oxidation, as described by Woodward et al., (1985). To ensure that the technique did not result in denaturation of protein epitopes, an additional electro-blotted membrane was subject to the same conditions but without addition of metaperiodate. Briefly, electro-blotted membranes were incubated in either 10mM sodium meta-periodate (in $50 \mathrm{mM}$ sodium acetate buffer, $\mathrm{pH} 4.5$ ) or just $50 \mathrm{mM}$ sodium acetate 
buffer, $\mathrm{pH} 4.5$ (for the controls), for one hour in the dark, followed by incubation with $50 \mathrm{mM}$ sodium borohydride in PBS for 30 minutes. After rinsing several times with PBS, the blots were blocked and developed in the same way as described above.

ImmunofluorescenceAdult, female Sprague Dawley rats ( 230g in weight) were given an overdose of pentobarbitone anesthetic $(0.5 \mathrm{ml} / 100 \mathrm{~g})$ via an intraperitoneal injection, and cardiac perfused with a $4.0 \%$ solution of paraformaldehyde (PFA) in Tris-buffered saline (TBS). The brain was removed and placed in PFA overnight. The following day specimens were transferred to a $30 \%$ sucrose solution in TBS, and allowed to sink fully in the solution at room temperature (RT). Subsequently, specimens were placed on a sliding, freezing microtome and $40 \mu \mathrm{m}$ coronal or sagittal sections cut through the brain from the most anterior or rostral section in the coronal plane in which the striatum can be recognised, to the cerebellum. Sections were placed in 24 well plates containing TBS with sodium azide, and 1 in 6 sections were taken for immunofluorescence using Py and NF-H antibodies.

For immunostaining, sections were rinsed 3 times in TBS, and subsequently incubated in a $0.5 \%$ Triton X-100 / TBS solution (TxTBS) for 1 hour at RT. Double-label antibody staining was conducted sequentially to avoid cross-reaction with secondary antibodies. Control sections were processed at the same time in the absence of either the Py or NF-H primary antibody, but included all steps containing secondary antibodies. Sections were first incubated overnight in a 1:500 dilution of NF-H (Sigma; N0142) in TxTBS. The following day, the sections were rinsed 3 times for 5 minutes in TBS and subsequently placed in a 1:500 dilution of Alexa Fluor ${ }^{\circledR} 594$ goat anti-mouse IgG secondary (Life Technologies) in TxTBS for 2 hours. Sections were subsequently rinsed 3 times for 20 minutes in TBS, and placed in a 1:50 dilution of Py antibody in TxTBS overnight. The following day sections were rinsed 3 times for 5 minutes, and subsequently incubated in a 1:500 dilution of Alexa Fluor ${ }^{\circledR} 488$ goat anti-mouse IgM (Life technology) and $0.5 \mu \mathrm{g} / \mathrm{ml}$ DAPI in TxTBS for 2 hours. Finally, the sections were rinsed 3 times for 20 minutes in TBS, and then mounted and coverslipped for microscopy. Images were captured using a Nikon Eclipse 80i microscope fitted with a Hammamatsu fluorescent camera operated with the NiS Elements computer software.

\section{$\underline{\text { Results }}$}




\section{Monoclonal antibody Py recognizes neurofilament heavy chain}

Immunoprecipitation from a rat cortex extract using Py MAb attached to protein-L, followed by mass spectrometry analysis of the captured material, resulted in the identification of several Py-antigen candidates (data not shown). Whilst some of these presumably bound to the protein-L non-specifically, only one candidate, neurofilament heavy chain (NF-H), corresponded to the approximate molecular weight of the major band recognised by Py MAb on a western blot ( 200kDa). Immunoprecipitations from a fresh extract of rat cortex using either Py MAb or an anti-NF-H MAb, followed by western blot analysis of the captured material, confirmed that immunoprecipitation and detection of the $\sim 200 \mathrm{kDa}$ antigen was reciprocal (Figure 1A). Control pull-downs, using cortex extract added to Dynabeads without antibody (for NF-H) and Protein-L without antibody (for Py), verified that the antigen did not bind to either matrix non-specifically (Figure 1A).

Py has historically been referred to as a "146 kDa glycoprotein" (Woodhams et al., 1989; Joosten et al., 2001; Houweling et al., 1999; Brook et al., 1997a; Brook et al., 1997b). To determine whether the epitope recognized by the Py antibody is glycosylated, electro-blotted membranes were subject to sodium metaperiodate treatment to destroy pentose and hexose carbohydrate epitopes by oxidation (Woodward et al., 1985). This treatment did not abolish immunoreactivity of the Py MAb, suggesting that the Py antibody epitope is unlikely to be glycosylated (Figure 1B).

The western blot band recognized by the Py and the NF-H MAb on eluates from both antibodies appeared to have a slightly slower electrophoretic mobility, compared to the unbound material following immunoprecipitation (Figure 1A). To confirm this, and to determine whether the antibodies immunoprecipitate a particular subset of the NF-H in the cortex extract, the eluates were subject to much longer separation on an SDS-PAGE gel, to better resolve higher molecular weight proteins before western blotting. Comparison of the material eluted from the Py and NF-H antibodies against the cortex extract before pull-down (i.e. "input") and the unbound material after pull-down revealed some interesting insights (Figure 1C and D). While the Py and the NF-H MAb both detected a sharp, well-resolved band of the same apparent molecular weight in the eluates, the NF-H MAb detected a band spanning a greater molecular weight range in the input and unbound material, compared to the Py MAb. Analysis of the same samples with an additional, commercially available 
antibody against NF-H, SMI-32, revealed the same pattern of immunoreactivity as seen with the NF-H antibody. Of note is the more discrete-sized band recognized by the Py MAb in the input and unbound (Figure $1 \mathrm{C}$ and D), and the clear distinction that the Py MAb makes between the faster electrophoretic mobility of the unbound material compared to the slower electrophoretic mobility of the protein that was immunoprecipitated (Figure 1C and D).

Taken together, these findings demonstrate that the Py and NF-H antibodies recognize the same sized isoform of NF-H in rat cortex RIPA extracts before protein denaturation, but following denaturation in SDS-sample buffer, a wider range of NF-H molecular weight forms - presumably varying in the degree of phosphorylation or other post-translational modification - are detectable by the NF-H MAb, compared to the Py MAb.

\section{Py and NF-H co-localize in the brain}

Immunofluorescencedouble-labelling with Py and NF-H antibodies yielded an overlapping staining pattern throughout the rat brain (Figures 2-5), while control sections (i.e., sections immuno-processed with both secondary antibodies, but excluding either of the primary antibodies) yielded labelling in only a single channel (Figure 6). DAPI labelling in each experimental condition indicates the presence of cells and confirms the integrity of the tissue. Co-localization of Py and NF-H in coronal sections from rostral regions of the brain (approximately $-0.12 \mathrm{~mm}$ from Bregma; Paxinos and Watson, 2005) was notable throughout the cortex, septal nuclei, and basal forebrain (Figure 2). While layer II / III and V of the cortex in this rostral plane showed obvious colocalization of Py and NF-H in neurons with large apical dendrites (Figures 2A-H), staining in the septal region was exclusively fibrous, and the basal forebrain a mixture of cellular and fibrous staining (Figures 2I-P). In the cortex, pyramidal cells in both layers II/III and V displayed intense staining of the cell body, proximal basal dendrites, as well as the full length of the apical process (Figures 2A-H; and 7A-D). Branching of the apical process in the most superficial regions of the cortex could be seen emanating from neurons in layer II/III. Fibers stained in the septal region (Figure 2I-L) were thick, though there was no obvious cell body staining to conclusively indicate that they were dendrites. In contrast, a small region of the basal forebrain showed a mixture of fiber (dendritic) and somatic staining (Figure 2M-P). 
Just caudal to this (approximately $-0.48 \mathrm{~mm}$ from Bregma; Paxinos and Watson), coronal sections show Py and NF-H colocalizing in the soma and apical dendrites of neurons in Layers II / III and V of the cortex (Figures 3A-H). However, though pyramidal neurons in the cingulate and somatosensory regions of the cortex were intensely immunopositive, the primary and / or secondary motor cortex appeared to have little or no immunoreactivity (Figures 3A-H). In this same plane of section, thick fiber staining highlighted the globus pallidus (Figures 3I-L) with both Py and NF-H antibodies, thoughit was not obvious whether these were dendritic or axonal. There was, however, a complete absence of fiber or cell body staining in the neighboring striatum..

Even more caudally (approximately $-3.48 \mathrm{~mm}$ from Bregma; Paxinos and Watson, 2005), coronal sections showed somatic and dendritic staining throughout the tightly packed CA3 region of the hippocampus (Figures 4A-H; 7E-H), and exclusively fibrous staining in the thalamus (Figures 4I-L). In the hippocampus, the soma of pyramidal neurons along with a short portion of their apical process were intensely Py/NF-H+ (Figure 7E-H). In both the thalamic and hippocampal region, staining in the red (NF-H) channel was occasionally more obvious (particularly in white matter tracts), compared to the staining seen with the Py antibody (green). This slight difference in antibody specificity may bear some relationship to the slightly narrower range of molecular weight forms that the Py antibody recognizes after protein denaturation by western blot, compared to the NF-H antibodies (Figure 1).

Caudally, in the cerebellum and brain stem (Figure 5), double labelling of Py and NF-H in sagittal sections showed staining in Purkinje cell soma and dendrites (Figures 5A-D), and in the soma of cells in the gigantocellular reticular formation (Figures 5E-L). Within the cerebellum, there was a notable absence of staining of any of the numberous neurons of the molecular and granuale cell layer (Figure 5A-D). Both the soma and proximal (thick) dendritic process of Purkinje cells were clearly Py/NF-H+, though the more fine structures of the dendritic tree were not obvious (Figures 5A-D; 7I-L). Again, staining with the NF-H antibody was more apparent in the white matter tract of the cerebellum in comparison to Py immunolabelling (Figure 5B-D). In the brain stem, the loosely packed group of neurons in the region of the gigantocellular reticular formation were intensely Py/NF-H+ (Figure 5E-L). Though fine tubular staining could be seen throughout the soma of the cells, there was little obvious neurite staining surrounding them (Figure 7M-P). 


\section{$\underline{\text { Discussion }}$}

Previously, the monoclonal antibody Py was shown to be useful for revealing intricate details of subsets of neurons within the brain and spinal cord. However, the identity of the antigen the antibody recognises remained a mystery. Here, immunoprecipitation from extracts of rat cortex, and subsequent immunofluorescencecharacterization shows that Py recognises the neurofilament heavy chain subunit.

In the original article detailing the production of the Py antibody, Woodhams et al (1989) describe how the antibody recognized a major band at $146 \mathrm{kDa}$ and a fainter band at $166 \mathrm{kDa}$ in various rat brain homogenates. However, in our hands, the major band recognized by the Py antibody has an apparent molecular weight of approximately $200 \mathrm{kDa}$, with some much fainter bands present at lower molecular weights (see Figure 1A). In contrast to the western blots shown here that were developed with an anti-IgM secondary antibody, Woodhams et al (1989) developed their Py blot using an anti-IgG secondary antibody that potentially lacked the required specificity to detect the (IgM-class) Py MAb. It is also possible that in previous attempts to resolve the Py antigen on an SDS-PAGE gel, the length of time the extracts were subject to electrophoresis was insufficient to enable the $200 \mathrm{kDa}$ protein to enter the resolving gel.

Py has historically been referred to as a "146 kDa glycoprotein" (Woodhams et al., 1989; Joosten et al., 2001; Houweling et al., 1999; Brook et al., 1997a; Brook et al., 1997b), presumably because it was a glycoprotein enriched fraction, isolated from a lentil lectinaffinity column, that was used for immunization (Woodhams et al., 1989). Although a glycosylated form of NF-H has been described previously (Cheung and Hart, 2008), our data suggest that the epitope that is recognized by the Py MAb is not glycosylated, since periodate treatment to destroy pentose and hexose carbohydrate epitopes by oxidation (Woodward et al., 1985) did not abolish immunoreactivity of the Py MAb (Figure 1). In the future, it will be interesting to determine whether differences in post-translational modification (e.g. phosphorylation and / or glycosylation) explain the narrower molecular weight range of NF$\mathrm{H}$ that is recognized by the Py MAb on western blots and whether this has any functional relevance. 
Morphologically, the cells that stain positive for Py/NF-H appear to have several characteristics in common. Neurons that express the antigen are large in size and many support large (typically apical) dendritic processes. This is illustrated well in layers II / III and V of the cortex (Figures 2-3), and in the CA3 region of the hippocampus (Figure 4). Within the cerebellum, there is a striking contrast between the immunoreactivity of the large cells in the Purkinje cell layer, and the lack of any detectable Py/ NF-H staining in the adjacent internal granule cell and molecular layers (Figure 5). In addition, the particularly large neurons in the gigantocellular reticular formation (Figure 5) are similarly immunopositive for Py/NF-H, while many brain stem neurons remain negative. Such observations are similar to work in the spinal cord by Brook et al., (1997a) who showed that only large diameter alpha motoneurons and the large neurons in Clarke's nuclei are positive for Py, while the small diameter neurons in the substantia gelatinosa are negative. This observation suggests that one possible explanation for the specific staining pattern of a subset of cells could be structural, in that the larger subunit of neurofilament may be important to the maintenance of the cytoskeletal framework of particularly large neurons. Indeed, work by Elder et al. (1998), have shown that mice with NF-H knocked out fail to develop large diameter axons in both the central and peripheral nervous system.

A notable exception to large diameter neurons staining positive for $\mathrm{Py} / \mathrm{NF}-\mathrm{H}+$ are the very large pyramidal neurons (particularly, Betz cells) in the motor cortex. Betz cells are among the largest neurons in the mammalian brain, yet they do not appear to stain positive for Py/NF-H. In an ultrastructural analysis of Betz cells in the human brain, Sasaki and Iwata (2001) have shown that neurofilament staining of these large neurons is rare, but that neurofilament accumulation is increased in Bunia-like bodies in Betz cells of human brains > 65 years of age and in neurons of the anterior horn of the spinal cord of ALS patients (Sasaki et al., 1989). Also, they observed that Betz cells appear to contain filaments that are much thicker than neurofilaments (i.e., 20-25nm in diameter). Such observations suggest that Betz cells have a particular cytoskeleton that is not dominated by a typical neurofilament structure, and that neurofilaments may mainly occur in these cells with increasing age or the presence of unusual cytoplasmic inclusions.

A second commonality among many of the cells and processes that stain positive for Py/NF$\mathrm{H}$, is that they reside in regions of the brain that appear to be particularly vulnerable to 
dementia or age related degeneration. The high expression of Py/NF-H in the cortex and CA3 hippocampal region, for example, correlates well with patterns of abnormal tau phosphorylation in Alzheimer's disease (Blazquez-Llorca et al., 2011). Furthermore, staining in the basal forebrain's substantia innominata likely include cells of the nucleus basalis of Meynert, which is known to be significantly affect by both Alzheimer's disease (AD) and Parkinson's disease (PD) (Liu et al., 2015). Similarly, connections to and from the cerebellum (Guo et al., 2016) and the septal region of the brain (Stroessner-Johnsson et al., 1992) are known to be affected by neurodegenerative disease or age related cell neuronal loss. Interestingly, seminal work by Chapman et al., (1989) showed that sera from AD patients showed a high content of antibodies directed again NF-H, and Roder and Ingram (1991) that age and AD- related changes in ATP may affect neurofilament/tau kinase activity.

Though these observations should not be overstated (as neurons in many regions of the CNS suffer age-related degeneration or are vulnerable to neurodegenerative disease - and the dopaminergic neurons of the substantia nigra pars compacta are not $\mathrm{Py} / \mathrm{NF}-\mathrm{H}+$ ) it seems important to continue to explore how large neurons may be more vulnerable to age related degeneration / disease (Mosconi et al., 2008), and whether Py/NF-H may provide a useful marker for these cells. Seminal work by Guo et al. (1995) and more recently by Veerana et al. (2011), in fact, strongly indicate that ageing may result in a hyperphosphorylation of NF-H; making cells more susceptible to degeneration. What is almost certain is that NF-H is known to be affected in (and therefore serve as a good marker for) a variety of neurodegenerative conditions. Work by Collard et al. (1995) provided some of the first evidence for NF-H accumulations being a factor in the degenerative process of amyotrophic lateral sclerosis (ALS). More recent work by Schulz et al. (2013) have shown that reduced phosphorylation of NF-H may be a contributing factor in neurofibromatosis type 2, and Sellner et al. (2014) that NF-H may be a useful pathological and prognostic marker for acute encephalitis. Interestingly, NF-H has also been shown to be a useful marker for the ganglion cells in the retina whose axons have been damaged (Drager and Hofbauer, 1984), as well regenerating fibers of retinal ganglion cells (Bates and Meyer, 1993).

In conclusion, the identification of the Py antigen sheds light on past work that has utilized the monoclonal antibody Py, and, when combined with studies using the NF-H antibody, expands our understanding of this protein in the developing, mature and dysfunction CNS. It will be important in the future to gain more understanding of why Py/NF-H expression in the 
brain appears mostly restricted to large diameter neurons, and whether this feature is relevant to neurodegenerative disorders and CNS injuries. The work presented here, and the collective work with Py and NF-H from previous studies, suggests a particular structure and function to cells which express this antigen in the brain and spinal cord, and illustrates how ultrastructural changes in these cells can be identified (in detail) in both normal and abnormal neural conditions.

\section{Compliance with Ethical Standards}

The authors declare that they have no conflict of interest. All applicable international, national, and/or institutional guidelines for the care and use of animals were followed. All procedures performed in studies involving animals were in accordance with the ethical standards of the institution or practice at which the studies were conducted. 


\section{$\underline{\text { References }}$}

Bates CA, Meyer RL (1993) The heavy neurofilament protein is expressed in regenerating adult but not embryonic mammalian optic fibers in vitro. Exp Neurol 119:249-257

Brook GA, Spitzer C, Nacimiento W, Kouchtir-Devanne N, Woodhams PL, Noth J (1997a) Differential distribution of immunoreactivity in the adult rat spinal cord revealed by the monoclonal antibody, Py: a light and electron microscopic study. Exp Neurol. 146:265-276

Brook GA, Nacimiento W, Taheri AS, Woodhams PL, Noth J (1997b) Axotomy-induced alterations in the red nucleus revealed by monoclonal antibody, Py, following a low thoracic spinal cord lesion in the adult rat. Spinal cord 35:474-481

Brook GA, Spitzer C, Nacimiento W, Woodhams PL, Noth J (1998) A novel early component of the cell body response in axotomized Clarke's nucleus neurons revealed by monoclonal antibody Py. Exp Neurol. 149:64-72

Blazquez-Llorca L, Garcia-Marin V, Merino-Serrais P, Avila J, DeFelipe J (2011) Abnormal tau phosphorylation in the thorny excrescences of CA3 hippocampal neurons in patients with Alzheimer's disease. J Alz Dis 26:683-698

Chapman J, Bachar O, Korczyn AD, Wertman E, Michaelson DM (1989) Alzheimer's disease antibodies bind specifically to a neurofilament protein in Torpedo cholinergic neurons. J Neurosci 9:2710-2717

Cheung WD and Hart GW (2008) AMP-activated protein kinase and p38 MAPK activate OGlcNAcylation of neuronal proteins during glucose deprivation. J Biol Chem 283:13009-20

Collard JF, Côté F, Julien JP (1995) Defective axonal transport in a transgenic mouse model of amyotrophic lateral sclerosis. Nature 375:61-64

Dräger UC, Hofbauer A (1984) Antibodies to heavy neurofilament subunit detect a subpopulation of damaged ganglion cells in retina. Nature 309:624-626 
Elder GA, Friedrich VL Jr, Kang C, Bosco P, Gourov A, Tu PH, Zhang B, Lee VM, Lazzarini RA (1998) Requirement of heavy neurofilament subunit in the development of axons with large calibers. J Cell Biol 143:195-205

Field PM, Seeley PJ, Frotscher M, Raisman G (1991) Selective innervation of embryonic hippocampal transplants by adult host dentate granule cell axons. Neurosci 41:713-727

Gou JP, Eyer J, Leterrier JF (1995) Progressive hyperphosphorylation of neurofilament heavy subunits with aging: possible involvement in the mechanism of neurofilament accumulation. Biochem Biophys Res Commun 15:368-376

Guo CC, Tan R, Hodges JR, Hu X, Sami S, Hornberger M (2016) Network-selective vulnerability of the human cerebellum to Alzheimer's disease and frontotemperal dementia. Brain [Feb 16. pii: aww003. [Epub ahead of print]]

Houweling DA, Brook GA, Gieling RG, Veldman H, Woodhams PL, Nacimiento W, Noth J, Bar PR, Joosten EA (1999) Differential distribution of immunoreactivity in the developing rat spinal cord revealed by the monoclonal antibody Py. Brain Res Dev Brain Res 116:87-96

Joosten EAJ, Van Westerlaak MGH, Biesheuvel C, Woodhams PL, Brook GA, Veldman H, Bar PR (2001) Cellular changes in motoneurons in a transgenic mouse model for amyotrophic lateral sclerosis as revealed by monoclonal antibody Py. Dev Brain Res 131:153-159

Lanier LM, Gates MA, Witke W, Menzies AS, Wehman AM, Macklis JD, Kwiatkowski D, Soriano P, Gertler FB (1999) Mena is required for neurulation and commissure formation. Neuron 22:313-325

Liu AK, Chang RC, Pearce RK, Gentleman SM (2015) Nucleus basalis of Meynert revisted: anatomy, history and differential involvement in Alzheimer's and Parkinson's disease. Acta Neuropathol 129:527-540 
Mosconi L, Pupi A, De Leon MJ (2009) Brain glucose hypometabolism and oxidative stress in preclinical Alzheimer's disease. Ann NY Acad Sci 1147:180-195

Paxinos G, Watson C (2005) The rat brain in stereotaxic coordinates, $5^{\text {th }}$ edition. Elsevier Acad Press

Roder HM, Ingram VM (1991) Two novel kinases phosphorylate tau and the KSP site of heavy neurofilament subunits in high stoichiometric ratios. J Neurosci 11:3325-3343

Sasaki S, Iwata M (2001) Ultrastructural study of Betz cells in the primary motor cortex of the human brain. J Anat 199:699-708

Sasaki S, Maruyama S, Yamane K, Sakuma H, Takeishi M (1989) Swellings of proximal axons in a case of motor neuron disease. Ann Neur 25:520-522

Schulz A, Baader SL, Niwa-Kawakita M, Jung MJ, Bauer R, Garcia C, Zoch A, Schacke S, Hagel C, Mautner VF, Hanemann CO, Dun XP, Parkinson DB, Weis J, Schröder JM, Gutmann DH, Giovannini M, Morrison H (2013) Merlin isoform 2 in neurofibromatosis type 2-associated polyneuropathy. Nat Neurosci 16:426-433

Sellner J, Davies NW, Howard RS, Petzold A (2014) Neurofilament heavy chain as a marker of neuroaxonal pathology and prognosis in acute encephalitis. Eur J Neurol 21:845-850

Shihabuddin LS, Brunschwig JP, Holets VR, Bunge MB, Whittemore SR (1996) Induction of mature neuronal properties in immortalized neuronal precursor cells following grafting into the neonatal CNS. J Neurocytol 25:101-111.

Stroessner-Johnson HM, Rapp PR, Amaral DG (1992) Cholinergic cell loss and hypertrophy in the medial septal nucleus of the behaviourally characterized aged rhesus monkey. $\mathrm{J}$ Neurosci 12:1936-1944

Veeranna, Yang DS, Lee JH, Vinod KY, Stavrides P, Amin ND, Pant HC, Nixon RA (2011)

Declining phosphatases underlie aging-related hyperphosphorylation of neurofilaments.

Neurobiol Aging 32:2016-2029 
Woodhams P, Webb M, Atkinson D, Seeley P (1989) A monoclonal antibody, Py, distinguishes different classes of hippocampal neurons J Neurosci 9:2170-2181

Woodward MP, Young WW Jr, Bloodgood RA (1985) Detection of monoclonal antibodies specific for carbohydrate epitopes using periodate oxidation. J Immunol Methods 78(1):143153 


\section{Figure legends}

Fig. 1 Reciprocal immunoprecipitation and western blot detection of Py and NF-H. (A) Western blot analysis of immunoprecipitates from a RIPA extract of rat cortex using either Py MAb or an anti-NF-H MAb confirmed that immunoprecipitation and detection of the $\sim 200 \mathrm{kDa}$ antigen was reciprocal. The control pull-down lanes refer to cortex extract incubated with Dynabeads without antibody (for NF-H) and Protein-L without antibody (for Py). The band identified by "*" is presumed to be a cross reaction of the anti-IgM secondary antibody with the IgM heavy chain that eluted from the Protein-L. The bands identified by ">" are presumed to be a minor cross-reaction of the Py or anti-IgM antibody with a component of the Protein- $\mathrm{L}$ that detached during elution of the immunoprecipitate in SDSsample buffer (B) Sodium meta-periodate treatment of electro-blotted membranes did not abolish immunoreactivity of the Py MAb. (C) Western blot analysis of the cortex extract, unbound extract (after pull-down) and eluates, following extended electrophoresis to separate higher molecular weight forms, is shown for the Py MAb immunoprecipitation and in (D) for the NF-H immunoprecipitation. Both sets of immunoprecipitates (C and $\mathbf{D})$ were also analyzed using another commercially available anti-neurofilament heavy antibody, SMI-32

Fig. 2 Immunofluorescence of coronal sections through the brain reveals colocalization of Py and NF-H in the cortex, septum, and basal forebrain. At approximately $-0.12 \mathrm{~mm}$ from bregma, Py and NF-H staining was evident in Layers II/III and V of the cingulate (A-D) and dorsal neocortex (E-H), as well as the fimbria (FI) and lateral septal (LS) regions (I-L), and the basal forebrain (M-P). While the soma (arrows B and F) and apical process (arrowheads B and F) of neurons in the cortex showed evident staining in layers II/III and V, there was only fibrous staining in the septal region, and a mixture of cell body and fiber staining in the basal forebrain. Scale bars in $A$ and $M=200 \mu \mathrm{m} ; \mathrm{E}=100 \mu \mathrm{m} ; \mathrm{I}=400 \mu \mathrm{m}$. (Diagram adapted from; Paxinos and Watson, 2005)

Fig. 3 Immunofluorescence of coronal sections through the brain reveals an absence of Py and NF-H staining in the motor cortex, and only fiberous staining in the globus pallidus. While the neurons in Layers II/III and V of the somatosensory (arrowhead 3B) and cingulate cortex (arrow 3B) stained intensely for Py and NF-H (A-H), there was a virtual absence of staining in much of the primary (M1) and secondary (M2) motor cortex (*3B), 
particularly in Layers II/III (A-H). Ventral to this, fibrous staining could be seen throughout the globus pallidus $(\mathrm{GP})$, though the whole of the striatum $(\mathrm{CPu})$ was immuno-negative for Py and NF-H (I-L). Scale bars in A and I $=400 \mu \mathrm{m} ; \mathrm{E}=200 \mu \mathrm{um} . \mathrm{M}=$ motor cortex. $\mathrm{Cg}=$ cingulate cortex. (Diagram adapted from; Paxinos and Watson, 2005)

\section{Fig. 4 Immunofluorescence of coronal sections through the brain reveals overlapping} staining for Py and NF-H in the hippocampus and thalamus. In caudal regions of the brain, Py and NF-H had completely overlapping staining in the CA3 region of the hippocampus (A-H), and reticular (rt) and ventral posteriormedial (VPM) and ventral posteriolateral (VPL) thalamic nuclei (I-L). Note that; although the cell soma and apical processes of neurons in the stratum lucidum (SLu) and CA3 regions of the hippocampus are labelled for Py and NF-H (E-H), only fibrous staining could be seen in nuclei of the thalamus (I-L). Scale bars in A and $\mathrm{I}=400 \mu \mathrm{m} ; \mathrm{E}=200 \mu \mathrm{m}$. (Diagram adapted from; Paxinos and Watson, 2005)

\section{Fig. 5 Immunofluorescence of sagittal sections through the brain reveals overlapping} staining for Py and NF-H in the cerebellum and brain stem. In caudal regions of the brain, Py and NF-H stained large cells of the Purkinje cell layer (PCL) of the cerebellum (AD) while the much smaller, but numerous, neurons of the internal granule cell (IGL) and molecular layer (ML) were negative. Similarly, in the brain stem, low (E-H) and high (I-L) powered images show that the very large cells of the gigantocellular group of the reticular formation (arrowheads J) are strongly positive for Py and NF-H staining. Boxed area in F, shown at higher magnification in I-J. Scale bars in A and E $=200 \mu \mathrm{m} ; \mathrm{I}=100 \mu \mathrm{m}$

\section{Fig. 6: Control staining for Py and NF-H show no cross reactivity with secondary}

antibodies. Low magnification $(1 \mathrm{x})$ image of coronal section of the rat brain double-labelled using Py (IgM) and NF-H (IgG) primary antibodies and corresponding anti-IgM (green) and anti-IgG (red) secondary antibodies (A-D). Inclusion of both primary and corresponding secondary antibodies reveal overlapping staining in the brain(D). In control sections, where the NF-H antibody was omitted (E-H), but both secondary antibodies were applied, no staining could be seen in the red channel (G). Similarly, in control sections where the Py antibody was omitted (I-L), but both secondary antibodies were applied, no staining could be seen in the green channel $(\mathrm{J}) . \mathrm{M}=$ motor cortex. $\mathrm{cc}=$ corpus callosum. ac $=$ anterior commissure. $\mathrm{CPu}=$ striatum. Scale bar in $\mathrm{A}=1 \mathrm{~mm}, \mathrm{E}=100 \mu \mathrm{m}, \mathrm{I}=200 \mu \mathrm{m}$ 
Fig. 7: High magnification, confocal images, showing cellular detail of Py/NF-H

colocalization: In layers II/III and V of the cortex, the soma, proximal basal dendrite (arrow head), and apical process (arrows) of pyramidal neurons were Py/NF-H+ (A-D). Similarly, in the tightly packed CA3 region of the hippocampus, the soma of large neurons and a short segment of their dendritic (apical) processes (arrow) could be seen in all pyramidal neurons. $(\mathrm{E}-\mathrm{H})$. In the cerebellum (I-L), it was notable that none of the vast number of cells in the granule cell layer (IGL), or the sparse cells of the molecular layer (ML) were immunopositive for Py/NF-H. By contrast, the monolayer of large Purkinje cells and their proximal dendritic tree were intensely $\mathrm{Py} / \mathrm{NF}-\mathrm{H}+$. In the brain stem (M-P), the sparse neurons of the gigantocellular reticular formation showed intense Py/NF-H staining of their soma, but little notable staining of neurite processes. Scale bars $=25 \mu \mathrm{m}$. 
A

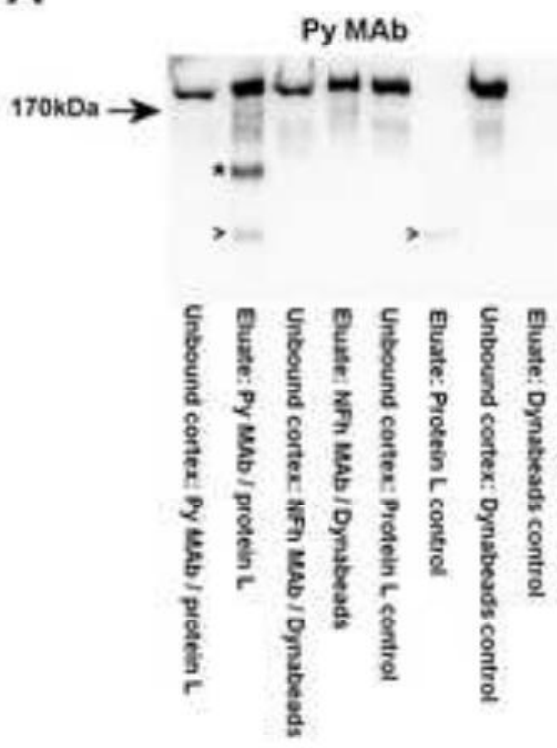

C

NF-H

phos + non phos
NF-H phos + non phos

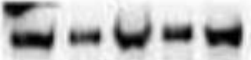

B

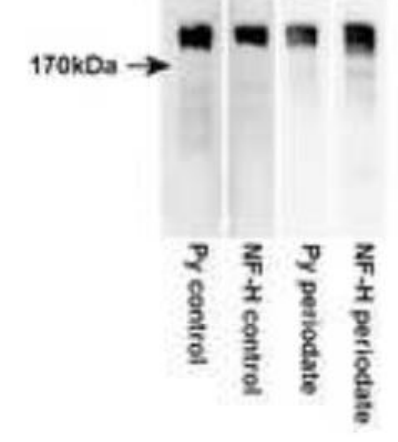

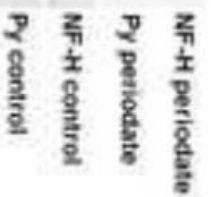

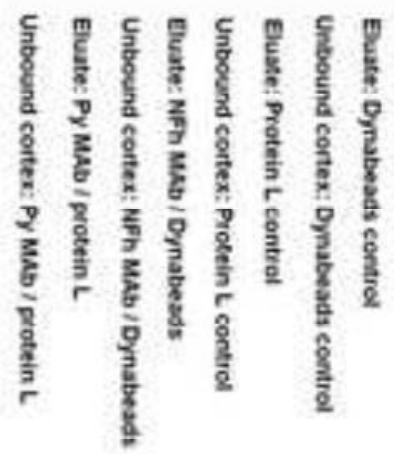

Py MAb

SMI-32

D

$$
\begin{gathered}
\text { NF-H } \\
\text { phos + non phos }
\end{gathered}
$$

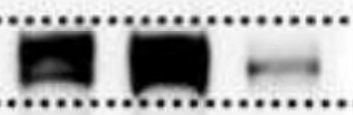

$170 \mathrm{kDa} \rightarrow$ 

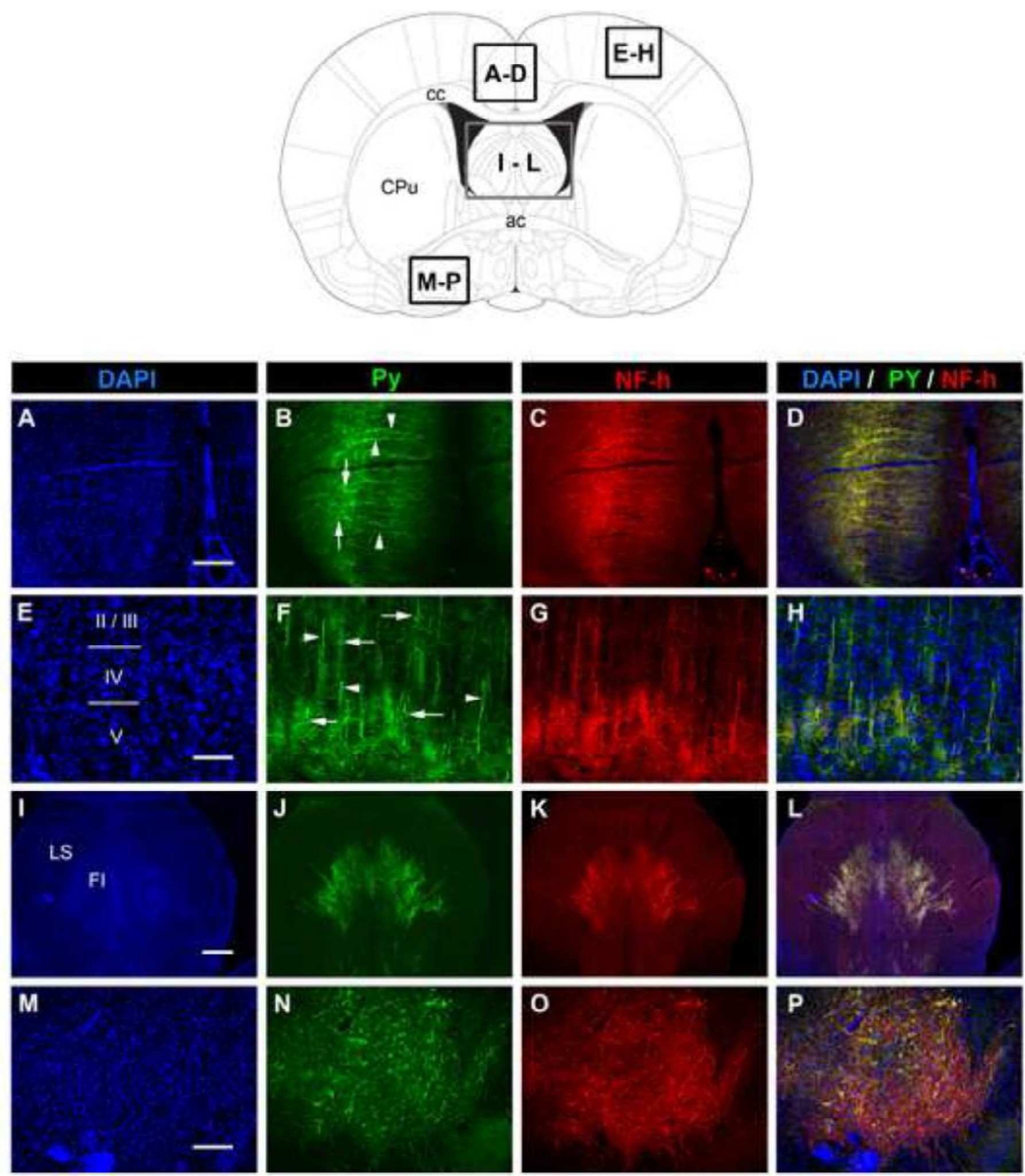

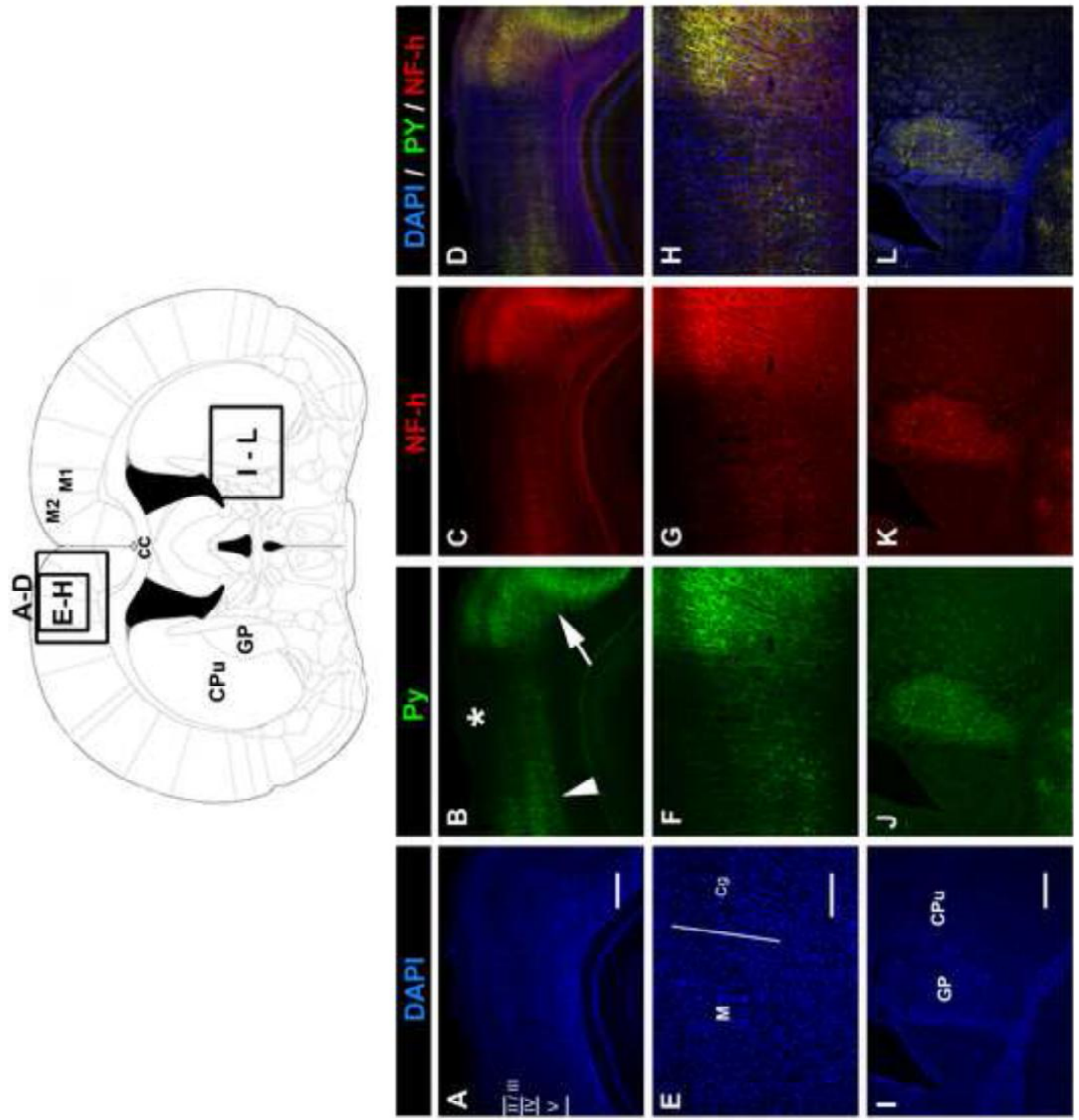

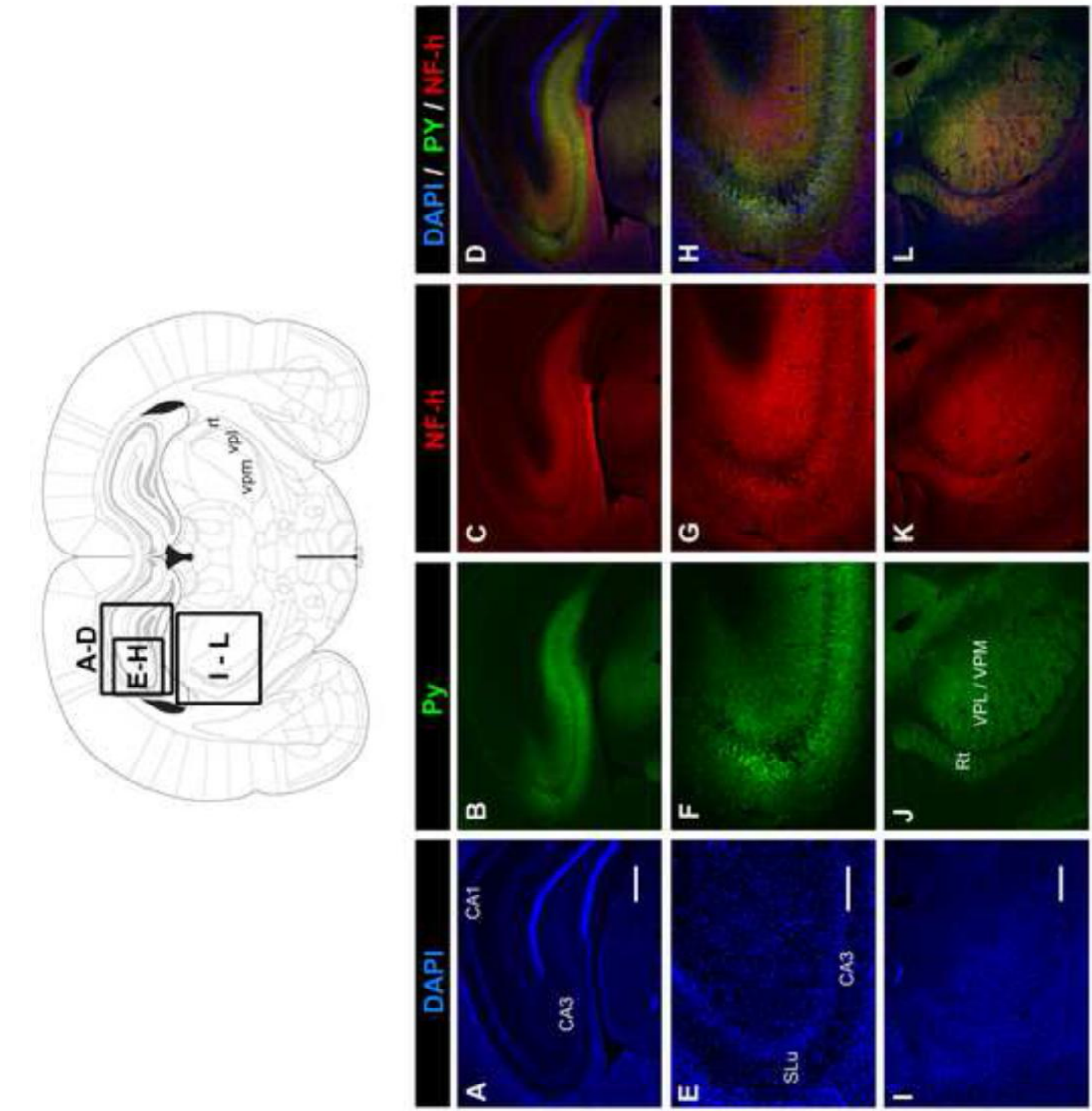

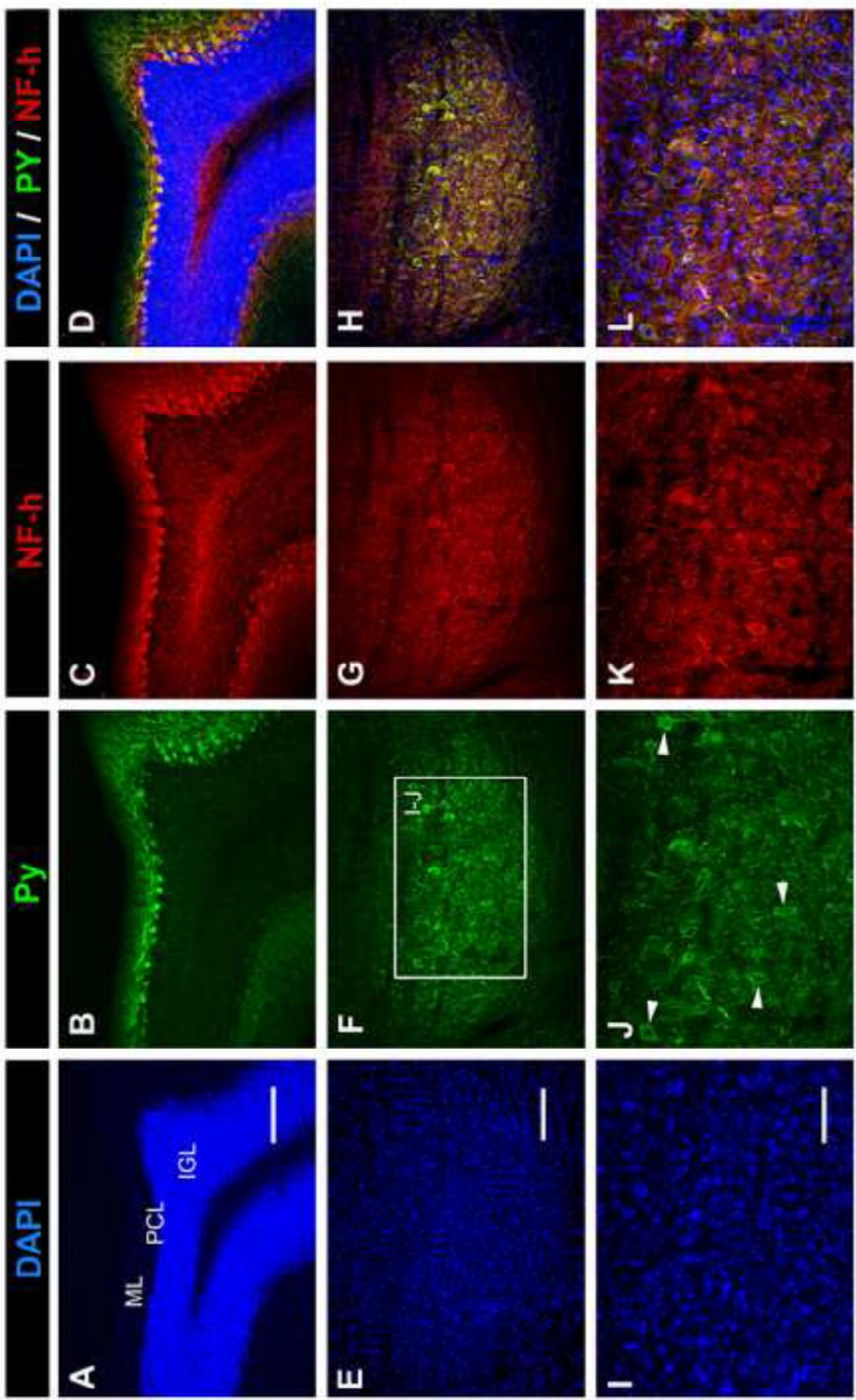

م) 

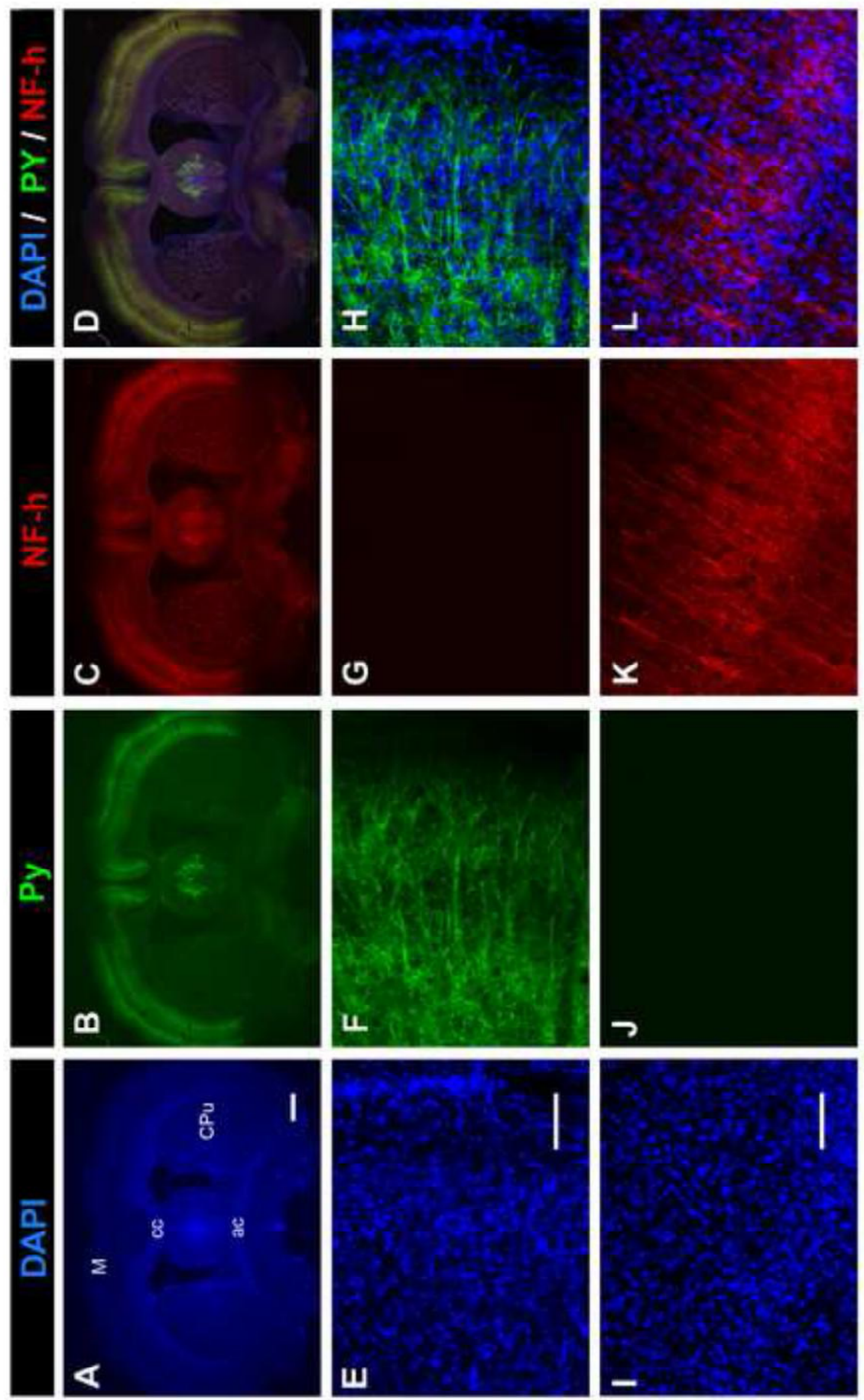

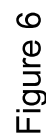



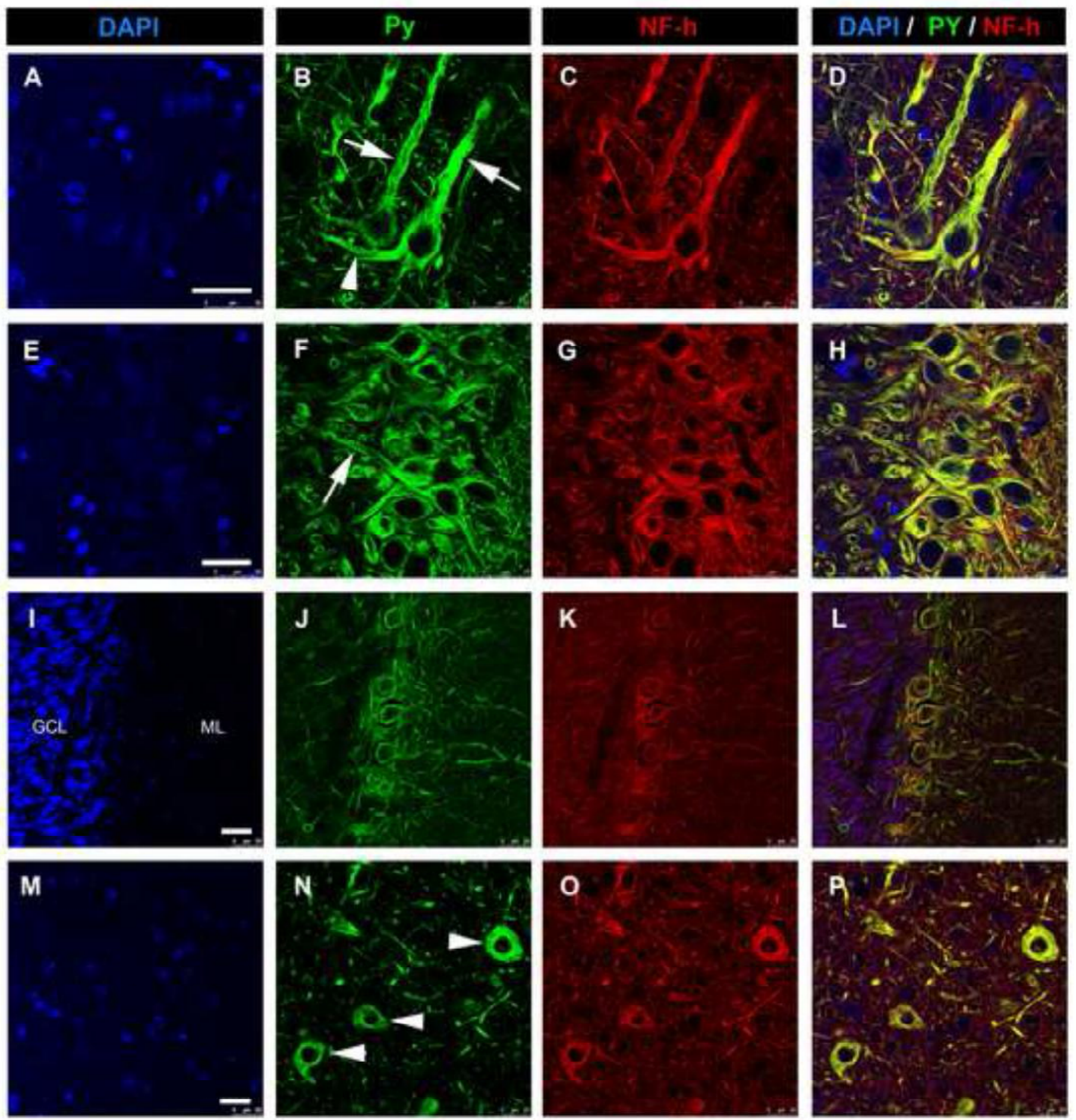